\title{
Expression of the human PAC1 receptor leads to dose-dependent hydrocephalus- related abnormalities in mice
}

\author{
Bing Lang, ${ }^{1}$ Bing Song, ${ }^{1}$ Wendy Davidson, ${ }^{1}$ Alastair MacKenzie, ${ }^{1}$ Norman Smith, ${ }^{2}$ \\ Colin D. McCaig, ${ }^{1}$ Anthony J. Harmar, ${ }^{3}$ and Sanbing Shen ${ }^{1}$

\begin{abstract}
${ }^{1}$ School of Medical Sciences, Institute of Medical Sciences, University of Aberdeen, Aberdeen, United Kingdom. ${ }^{2}$ Ultrasound Department, Aberdeen Maternity Hospital, Grampian University Hospitals NHS Trust, Aberdeen, United Kingdom.
\end{abstract} \\ ${ }^{3}$ Centre for Neuroscience Research, School of Biomedical Sciences, University of Edinburgh, Edinburgh, United Kingdom.
}

\begin{abstract}
Hydrocephalus is a common and potentially devastating birth defect affecting the CNS, and its relationship with G protein-coupled receptors (GPCRs) is unknown. We have expressed 2, 4, or 6 copies of a GPCR - the human PAC1 receptor with a 130-kb transgene in the mouse nervous system in a pattern closely resembling that of the endogenous gene. Consistent with PAC1 actions, PKA and PKC activity were elevated in the brains of Tg mice. Remarkably, Tg mice developed dose-dependent hydrocephalus-like characteristics, including enlarged third and lateral ventricles and reduced cerebral cortex, corpus callosum, and subcommissural organ (SCO). Neuronal proliferation and apoptosis were implicated in hydrocephalus, and we observed significantly reduced neuronal proliferation and massively increased neuronal apoptosis in the developing cortex and SCO of Tg embryos, while neurite outgrowth and neuronal migration in vitro remain uncompromised. Ventricular ependymal cilia are crucial for directing cerebrospinal fluid flow, and ependyma of Tg mice exhibited disrupted cilia with increased phospho-CREB immunoreactivity. These data demonstrate that altered neuronal proliferation/apoptosis and disrupted ependymal cilia are the main factors contributing to hydrocephalus in PAC1-overexpressing mice. This is the first report to our knowledge demonstrating that misregulation of GPCRs can be involved in hydrocephalus-related neurodevelopmental disorders.
\end{abstract}

\section{Introduction}

Hydrocephalus and neural tube defects (NTDs) are devastating birth defects affecting the CNS and are common worldwide. Hydrocephalus accounts for approximately $40 \%$ of these CNS abnormalities, and its causes remain obscure.

In mice, genetic alterations of signal transduction components are associated with CNS malformation. For example, Socs7 is a member of the suppressor of cytokine signaling family and regulates receptor tyrosine kinases. Around $50 \%$ of Socs $7^{-/-}$mutant mice die within 15 postnatal weeks with hydrocephalus, thinned cerebral cortex, and disorganized subcommissural organ (SCO) $(1,2)$, all of which are commonly associated with human hydrocephaly (3). PKC is involved in neural induction, and mice lacking PKC substrates myristoylated, alanine-rich C-kinase substrate (MARCKS), MARCKS-related protein, or p190 RhoGAP develop NTDs and hydrocephalus-related phenotypes, such as enlarged lateral ventricles (LVs), agenesis of the corpus callosum (cc), and lateral displacement of the forebrain (4-6). Specific PKC isoforms are required for the prevention of NTDs in curly tail mice (7). Interestingly, increased PKC and diacylglycerol activities are also associated with NTDs in a diabetic model (8). PKA is equally crucial

Nonstandard abbreviations used: $c c$, corpus callosum; CREB, cAMP response element-binding protein; CSF, cerebrospinal fluid; GPCR, G protein-coupled receptor; Het, heterozygous; IFT, intraflagellar transport; IRES, viral internal ribosomal entry site; LV, lateral ventricle; MARCKS, myristoylated, alanine-rich C-kinase substrate; NTD, neural tube defect; OLP, oligodendrocyte progenitor; PAC1, PACAP type I; PACAP, pituitary adenylate cyclase-activating polypeptide; phospho-CREB phosphorylated CREB; SCO, subcommissural organ; STS, sequence-tagged site. Conflict of interest: The authors have declared that no conflict of interest exists. Citation for this article: J. Clin. Invest. 116:1924-1934 (2006). doi:10.1172/JCI27597. for CNS development. PKA-null mutants are embryonic lethal, and mice with one-quarter of PKA activity show NTDs with a significantly enlarged lumen (9). PKA can phosphorylate and activate cAMP response element-binding protein (CREB). Significantly, $\mathrm{CREB}^{-/-}$mice develop complications including dilated LVs and severely reduced $\mathrm{cc}$ and anterior commissures (10). Although many of the signal transduction components are implicated in CNS abnormalities, the identity of upstream components such as transmembrane receptors and ligands remains elusive.

We are interested in G protein-coupled receptors (GPCRs) for the neuropeptides pituitary adenylate cyclase-activating polypeptide (PACAP) and vasoactive intestinal peptide (VIP), for which 3 receptors (VPAC1, VPAC2, and PAC1) are identified (11). VPAC1 and VPAC2 receptors are equally sensitive to both peptides. Previously, we discovered that the VPAC2 receptor is essential for circadian function $(12,13)$. However, the PACAP type I (PAC1) receptor is selectively activated by PACAP, and its mRNA is expressed predominantly in the CNS (14). In mouse embryo, high levels of PAC1 mRNA are detected in the floor and roof plates of the neural tube (15), suggesting that PACAP plays important roles in the early development of the CNS.

Clinical evidence suggests that gain-of-function of chromosomal regions including genes in the PACAP signaling pathway leads to neurodevelopmental disorders. The PACAP gene (ADCYAP1) resides at $18 \mathrm{p} 11$. Fetuses with trisomy 18 develop microcephaly and spina bifida (16), and patients with tetrasomy 18p suffer from microcephaly, mental retardation, and congenital hydrocephalus (17). The $18 \mathrm{p} 11$ region is also associated with an increased susceptibility to schizophrenia (18), and dilation of the LVs is a prominent anatomical feature in schizophrenia. The PAC1 gene 


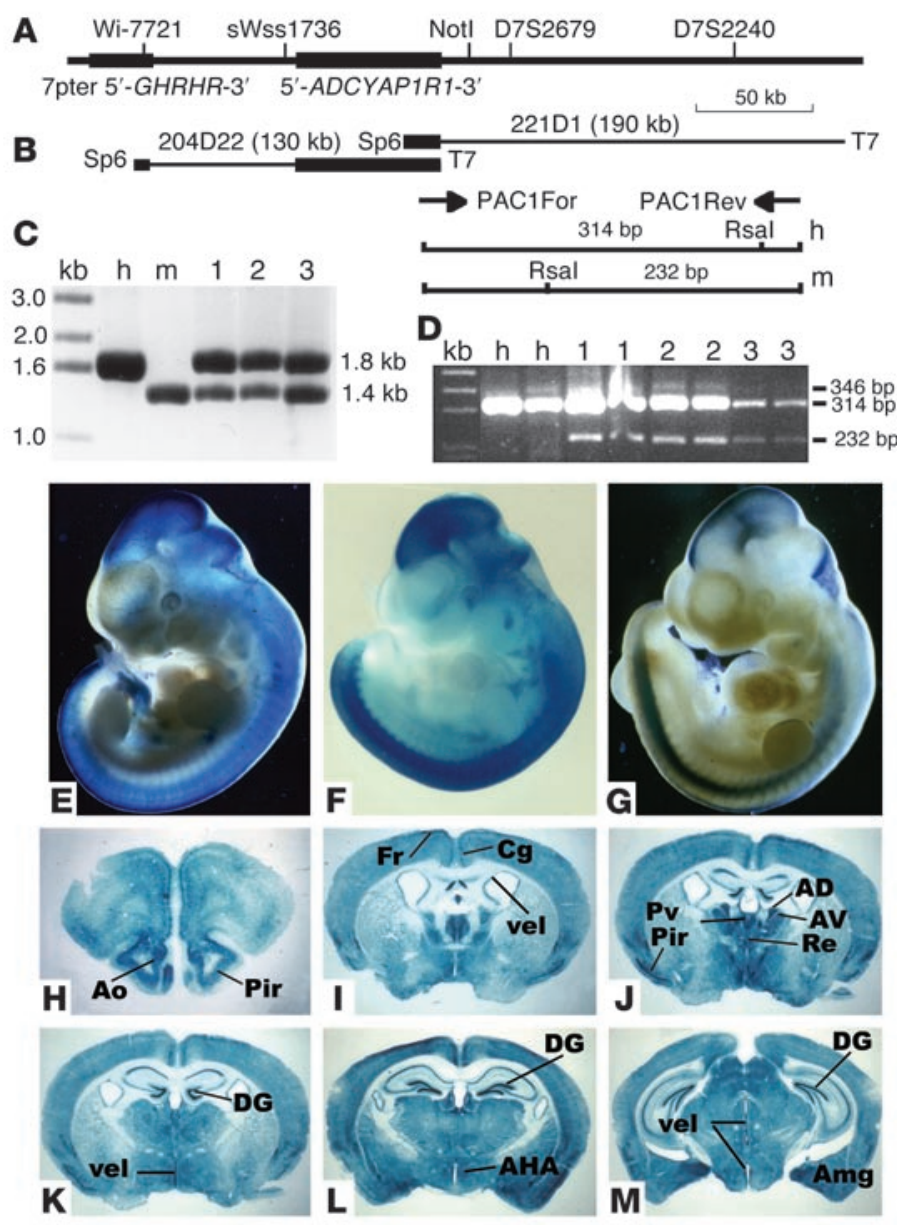

\section{Figure 1}

Genomic organization and expression of the human ADCYAP1R1 transgene. (A) Mapping of the human ADCYAP1R1 gene encoding the PAC1 receptor to $7 p$ between STS markers sWss1736 and D7S2679. (B) Overlapping of 221D1 with 204D22 at the $3^{\prime}$ half of the PAC1 receptor coding region. (C) Determination of transgene copy number by semiquantitative PCR with primers PAC1For and PAC1Rev. The ratios of the 1.8-kb band (human) to the 1.4-kb fragment (mouse) indicated that Tg1 (lane 1), Tg2 (lane 2), and Tg3 (lane 3) contained 6, 4, and 2 copies of the transgene, respectively. (D) The expression of the human PAC1 mRNA in E10.5 mouse embryos detected by RT-PCR with the primers PAC1For and PAC1Rev. Rsal restriction digestion of the PCR products resulted in 314-bp and 232-bp bands from human and mouse PAC1 mRNA, respectively. Human fetal CDNA (h) was included as a control. m, mouse. (E-G) X-gal staining of E10.5 mouse embryos derived from lines Tg1 (E), Tg2 (F), and Tg3 (G), revealing transgene expression in the nervous system. $(\mathbf{H}-\mathbf{M})$ X-gal staining of coronal sections of adult brains, showing high levels of transgene expression in anterodorsal thalamic nucleus $(A D ; J)$, anterior hypothalamic nucleus (AHA; L), amygdala (Amg; $M)$, anterior olfactory nucleus (Ao; $\mathbf{H})$, anteroventral thalamic nucleus (AV; J), cingulate cortex (Cg; I), dentate gyrus (DG; K and $\mathbf{M})$, frontal cortex (Fr; I), piriform cortex (Pir; J), paraventricular nucleus of the thalamus $(\mathrm{Pv} ; \mathbf{J})$, reuniens thalamic nucleus (Re; J), and ventricular ependymal layer (vel; I, K and M).
(ADCYAP1R1) is situated at 7p15. A patient with 7p15 duplication exhibits severe mental deficiency with communicating hydrocephalus (19). Indisputably, these chromosomal regions encode a large number of genes; however, this evidence collectively hints that elevated PACAP signaling through the PAC1 receptor may contribute to some of these neurologic manifestations.

To investigate the role of the PAC1 receptor in this regard, we have generated $\mathrm{Tg}$ mice overexpressing the human PAC1 receptor encoded by a $130-\mathrm{kb}$ P1 bacterial artificial chromosome (PAC). The PAC was modified (20) to coexpress PAC1 and a lacZ reporter (with the use of a viral internal ribosomal entry site [IRES]) in the nervous system. Significantly, Tg mice developed hydrocephalusrelated phenotypes in a dose-dependent manner.

\section{Results}

Genomic organization of the buman ADCYAP1R1 gene. Two positive clones (204D22 and 221D1) were identified from the RPCI-1 Human PAC Library (21) as containing the human ADCYAP1R1 gene (Figure 1B). The $130-\mathrm{kb}$ clone 204D22 comprises the entire gene ( 50 $\mathrm{kb}$ ) and approximately $70 \mathrm{~kb}$ of upstream sequence with sequencetagged site (STS) markers Wi-7721 and sWss1736. The 190-kb clone 221D1 overlaps with 204D22 at the $3^{\prime}$ half of the ADCYAP1R1 gene and harbors D7S2679 and D7S2240 (Figure 1A).

We subcloned and sequenced the T7 ends of both clones. The end sequences and STS markers were used for DNA database searching. Three overlapping genomic segments (GenBank acces- sion numbers AC005155, AC006466, and AC006398) were identified, enabling construction of a 356-kb contig flanking the human ADCYAP1R1 gene. 204D22 was found to start with Wi-7721 at nt 65,850 of AC005155 and to terminate at nt 70,660 of AC006466; while 221D1 ended at nt 72,954 of AC006398. The marker Wi-7721 was derived from the 3'UTR of the gene encoding growth hormone-releasing hormone receptor (GHRHR), whose ligand belongs to the same neuropeptide superfamily including PACAP, suggesting that the ADCYAP1R1 and GHRHR genes may have evolved from a common ancestor.

The human PAC1 transgene and expression. We chose 204D22 to overexpress the human PAC1 receptor in mice, as bioinformatic analysis revealed that it encoded only the PAC1 receptor but no other functional genes (data not shown). To facilitate detection of transgene expression, we incorporated an IRES-lacZ reporter gene at the 3'UTR. The modified PAC DNA was introduced into fertilized eggs by pronuclear microinjection. Of 67 offspring, 9 founders were identified to integrate the intact transgene, and $8 \mathrm{Tg}$ lines were established, with the exception of the highest expressing founder.

The transgene copy number was determined by semiquantitative PCR, with primers (PAC1For and PAC1Rev) from exons 7 and 9 that are completely conserved between human and mouse. Ratios of the intensity of a $1.8-\mathrm{kb}$ product (from the transgene) to that of a 1.4-kb band (from the endogenous gene, 2 copies) were quantified using NIH Image. Tg1, Tg2, and Tg3 lines were 


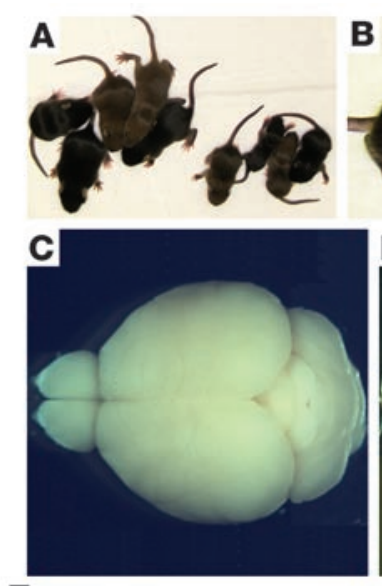

E
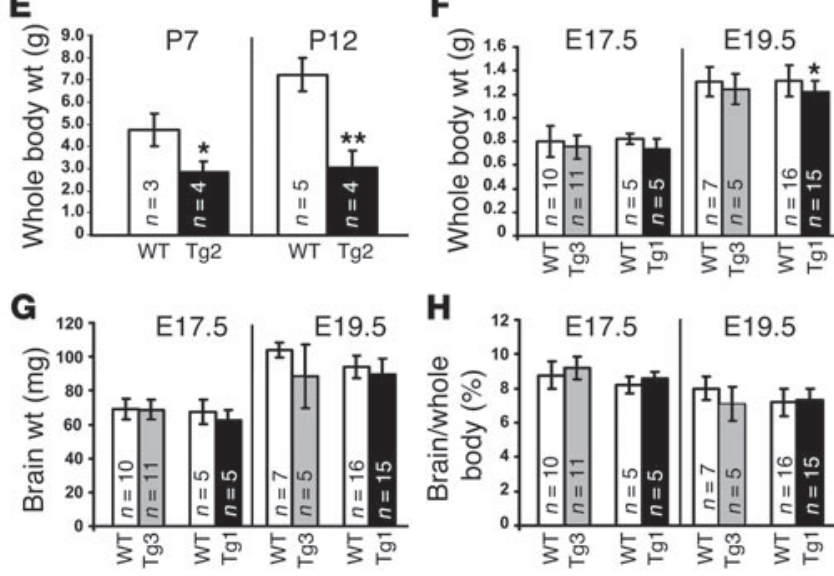

found to carry 6,4 , and 2 copies of the transgene, respectively, and characterized in detail (Figure 1C).

The transgene expression was analyzed by X-gal staining, which showed integration site-independent expression in the nervous system (Figure 1, E-G), closely resembling the endogenous expression pattern (15). Intense lacZ staining was observed in the roof and floor plates of the neural tube, the hindbrain, midbrain, and diencephalons, and the dorsal root ganglia. In adult brain, the lac Z expression patterns largely reproduced that of rodent $P A C 1 \mathrm{mRNA}$ (14), with widespread expression in the CNS (Figure 1, H-M). The highest expression level was detected in the hippocampus, especially in granule cells of the dentate gyrus and pyramidal cells in the CA1-CA3 regions (Figure 1, K-M). Other prominent expression sites included cerebral cortex (especially the piriform, cingulate, and frontal cortices; Figure 1, I and J), the hypothalamus (especially the anterior hypothalamic area; Figure 1L), thalamic nuclei (including the anterodorsal, anteroventral, paraventricular, and reuniens nuclei; Figure 1J), the anterior olfactory nucleus (Figure $1 \mathrm{H}$ ), and the external granule cell layer of the cerebellum (data not shown). Strong $\beta$-gal activity was also observed in the ventricular ependymal layer (Figure 1, I, K, and M), the dorsal horn and central canal of spinal cord (Supplemental Figure 1; supplemental material available online with this article; doi:10.1172/JCI27597DS1), the adrenal medulla (Supplemental Figure 1), and the inner nuclear layer and ganglion cell layer of retina (data not shown).

To reveal the relative abundance of the transgene expression, we carried out RT-PCR with E10.5 RNA and primers PAC1For and

\section{Figure 2}

Growth retardation and hydrocephalus in PAC1-overexpressing mice. (A) A litter of P12 Tg3 heterozygote $\times$ heterozygote mice showed marked variation in body size. (B) The smallest littermate (right) weighed approximately a quarter of the normal weight (left) and displayed a mildly dome-shaped skull, typical of a progressive hydrocephalus phenotype. (C and $\mathbf{D}$ ) Images of the same magnification of brains were taken from a 5-week-old WT (C) and a Tg3 homozygotic littermate (D), and the latter manifested severe hydrocephalus with unfused frontal cortex (thin arrow) and dramatically thinned occipital and parietal cortex (thick arrows). (E) The growth of Tg2 mice was significantly retarded by P7 and P12. (F) Tg1 but not Tg3 newborns were significantly smaller than their WT littermates at E19.5; however, the difference was marginal at E17.5. Neither Tg3 nor Tg1 transgenics differed significantly from their WT littermates in brain weight $(\mathbf{G})$ or ratios of brain to whole-body weight $(\mathbf{H})$ at E17.5 or E19.5. ${ }^{\star} P<0.05$; ${ }^{* \star} P<0.01$.

PAC1Rev. The ratios of a 314-bp RsaI fragment (from human PAC1 mRNA) to a 232-bp RsaI band (from mouse endogenous mRNA) confirmed transgene copy number-dependent expression of the human PAC1 receptor in all $3 \mathrm{Tg}$ lines (Figure 1D).

Transgene dosage-dependent pre-weaning loss. The Tg founder (male) with the highest transgene copy number produced Tg embryos but no viable Tg offspring, suggesting that a high level of PAC1 receptor expression could cause embryonic or early postnatal death. This was supported by other genotyping data in heterozygous (Het) $\times$ WT breeding. The offspring of Tg 3 mice with 2 copies of the transgene exhibited a typical Mendelian ratio (41 Tg3/45 WT), while only $58 \%$ of Tg2 (4 copies, $110 \mathrm{Tg} 2 / 191 \mathrm{WT}, \sim 6.1$ mice/litter) and $51 \%$ of $\operatorname{Tg} 1$ ( 6 copies, $87 \mathrm{Tg} 1 / 172 \mathrm{WT}, \sim 6.6$ mice/litter) heterozygotes survived to the weaning stage. Breeding records revealed $13 \%$ postnatal loss within the first week in $\operatorname{Tg} 1$ and $\operatorname{Tg} 2$ lines. To determine possible embryonic lethality, 5 litters totaling 31 pups from $\mathrm{Tg} 1 \times \mathrm{WT}$ mice $(6.2 \mathrm{mice} / \mathrm{litter})$ were dissected at E19.5 right before natural birth, and 15 of them were $\mathrm{Tg}$ (Figure $2 \mathrm{~F}$ ), showing no significant loss of $\mathrm{Tg}$ fetuses before birth.

At the weaning stage, $\operatorname{Tg} 1$ and $\operatorname{Tg} 2$ mice were notably smaller than WT littermates. To determine the timing of growth retardation, we analyzed P12, P7, E19.5, and E17.5 littermates (Figure 2). The Tg2 heterozygotes were significantly lighter than WT littermates by P7 $(P<0.02)$ and P12 $(P<0.001)$. However, at E17.5 or E19.5, their differences were marginal. Therefore, growth retardation largely resulted from disadvantaged postnatal development.

Tg3 homozygotes also showed profound growth retardation (Figure 2B, right). Similar to $\operatorname{Tg} 1$ and $\mathrm{Tg} 2$ heterozygotes, a small proportion (5\%) of Tg3 homozygotes developed a dome-shaped skull (Supplemental Figure 2), with excess cerebrospinal fluid (CSF), unfused frontal cortex (thin arrow, Figure 2D), and remarkable thinning of occipital and parietal cortex (thick arrows, Figure 2D). However, the majority of Tg mice did not display the typical dome-shaped skull, suggesting that only a small proportion of $\mathrm{Tg}$ mice developed overt hydrocephalus before fusion of the sutures.

Transgene dosage-dependent hydrocephalus-related phenotypes. To examine anatomic changes in the CNS of Tg mice, we processed histologically 8 brains of each Tg line and 8 brains of WT littermate adults. Morphological quantification of 14 brains (5 WT [Figure $3, \mathrm{~A}$ and $\mathrm{F}$ ] and 3 of each $\mathrm{Tg}$ line [Figure $3, \mathrm{~B}$ and $\mathrm{G}$ ]) showed a clear transgene dosage-dependent dilation of the LVs (Figure 3, C and $\mathrm{H}$ ) in Tg mice compared with WT littermates. In addition, the 
A

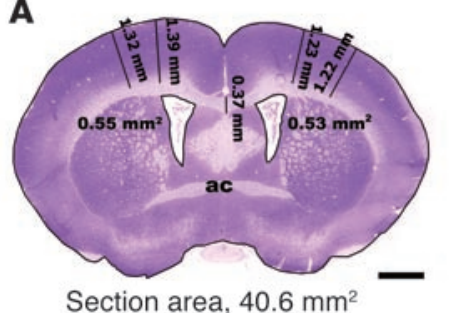

B
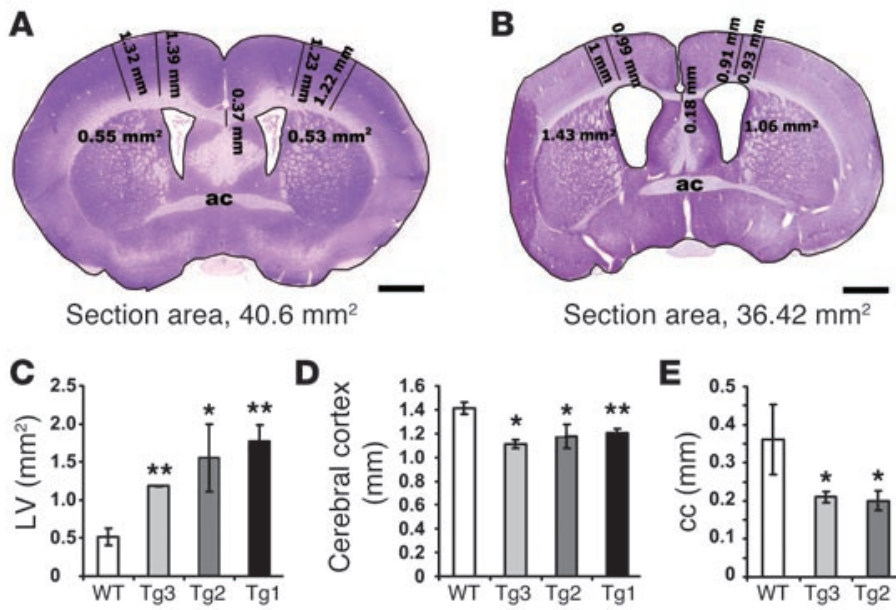

D

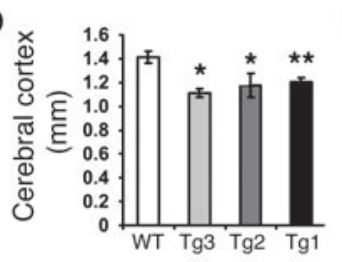

E

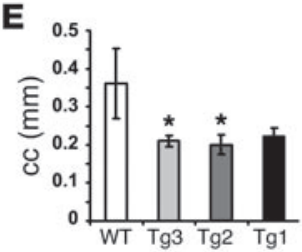

$\mathbf{F}$

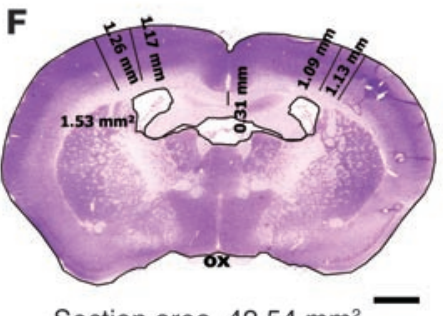

Section area, $42.54 \mathrm{~mm}^{2}$

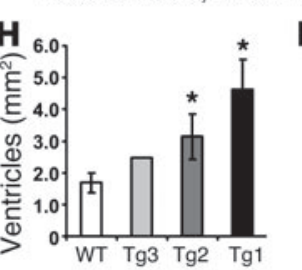

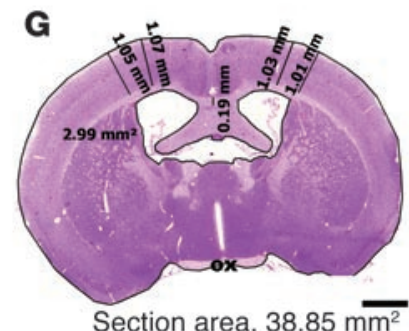

J

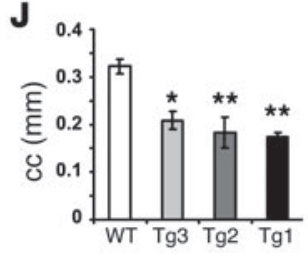

Figure 3

Morphometric evidence showing dose-dependent hydrocephalus in PAC1 Tg mice. (A, B, F, and $\mathbf{G})$. Coronal sections of adult brains from WT (A and $\mathbf{F})$ and $\mathrm{Tg}$ (B and $\mathbf{G})$ mice were stained with cresyl violet. The sizes of ventricles ( $\mathbf{C}$ and $\mathbf{H}$ ), the thickness of the cerebral cortex ( $\mathbf{D}$ and $\mathbf{I}$ ) and of the cc ( $E$ and $\mathbf{J}$ ), as well as the section area were quantified by AxioVision Rel. 4.5 (Zeiss) at comparable planes across the anterior commissure (ac; $\mathbf{A}$ and $\mathbf{B}$ ) or optic chiasm (ox; $\mathbf{F}$ and $\mathbf{G}$ ) from 5 WT and $3 \mathrm{Tg}$ mice of each line. Note transgene dosage-dependent dilation of the LVs (C and $\mathbf{H}$ ) and significant reduction of the cerebral cortex (D and $\mathbf{I})$ and $c c(\mathbf{E}$ and $\mathbf{J}) .{ }^{*} P<0.05$; ${ }^{* *} P<0.01$. Scale bars: $1 \mathrm{~mm}$ in $\mathbf{A}, \mathbf{B}, \mathbf{F}$, and $\mathbf{G}$.

offspring of $\mathrm{Tg} 3$ mice histologically and found a number of abnormalities. In WT littermates, the hippocampus appeared caudally to the septum, while hippocampus of $\mathrm{Tg}$ mice was remarkably rostralized and became visible before the merger of the bilateral septum (Figure 5B). In addition, it was displaced laterally from the midline (Figure 5, D, F, and H) and developmentally retarded (see Figure 5, $\mathrm{G}$ and $\mathrm{H}$, for comparison). The cc was underdeveloped (see Figure 5, C and D), and, consequently, the cerebral hemispheres of $\mathrm{Tg}$ mice were dislocated laterally, with a widened longitudinal fissure (arrows, Figure 5, B, D, F, and H). However, at E19.5 brains of Tg fetuses did not differ significantly from those of WT littermates in size, weight, or ratios of brain to whole body weight (Figure 2, F-H). The LVs of Tg mice were mildly enlarged (Figure 5, B and D) compared with those of WT littermates (Figure 5, A and C), suggesting that overt dilation of the ventricles in $\mathrm{Tg}$ adults largely developed after birth.

Reduced thickness of cerebral cortex and cc. The cc consists of nerve fibers projecting from cortical neurons to comcerebral cortex and $c c$ were also significantly thinned in brains of Tg mice (Figure 3, D, E, I, and J).

Ventricular ependymal cells are important for CSF circulation. High levels of transgene expression were observed in the ependyma of the LVs (Figure 1I), third ventricle (Figure 4, D and L), and aqueduct (Figure $4 \mathrm{H}$ ). The ependymal lining of the ventricles seemed intact in the $\mathrm{Tg}$ mice. However, the third ventricle was also expanded in all Tg lines (Figure 4, B-D), and the aqueduct was enlarged and grossly deformed (Figure 4, F-H). The SCO is crucial for CSF flow through the aqueduct. Misexpression or deletion of transcription factors such as Engrailed 1, RFX4, and Msx1 results in hydrocephalus associated with SCO malformation (22-24). The PAC1 transgene was expressed strongly in the SCO (Figure 4L), and the sizes of SCO (Figure 4, J-L) and the numbers of SCO cells were significantly reduced in $\mathrm{Tg} 1$ and $\mathrm{Tg} 2$ mice (Figure $4 \mathrm{M} ; n=3$ each).

In patients, spina bifida is commonly associated with hydrocephalus. The PAC1 transgene was highly expressed in the developing neural tube (Figure 1, E-G) and adult spinal cord (Supplemental Figure 1). However, we did not observe failure of neural tube closure in $\mathrm{Tg}$ heterozygotes at any stage. Therefore, the hydrocephalus-related phenotypes are unlikely to be caused by defects in neural tube closure.

Congenital brain defects in the PAC1 Tg newborns. To investigate possible congenital abnormalities, we analyzed newborn (P1) brains from Het $\times$ WT offspring of $\mathrm{Tg} 1$ and $\mathrm{Tg} 2$ lines and from Het $\times$ Het municate between the 2 hemispheres. Thinned cc and cerebral cortex were commonly present in all $\mathrm{Tg}$ adults (Figure 3, D, E, I, and $\mathrm{J}$ ), indicating possible agenesis of the cc. In Tg newborns, they were also substantially reduced (Figure 5 and Figure $6 \mathrm{E}$ ). In contrast, the cc was well formed and projected to the contralateral side in WT littermates (cc; Figure 5C and Figure 6D). Examination of serial Nissl-stained sections revealed that the cc was present only in a short medial region of $\mathrm{Tg}$ mouse brains, with a reduced thickness (Figure 6E), while in more rostral or caudal regions, it failed to cross the midline but formed large ipsilateral whorls of callosal axons known as Probst bundles (p; Figure 5, B and D). The development of anterior and posterior commissures was abnormal in some other hydrocephalic mice $(10,22,24)$; however, they remained anatomically normal in our Tg mice (Figures 3B and Figure 4, J-L).

Two populations of midline glial cells, the glial wedge and the indusium griseum, are critical for axon guidance of cortical neurons and callosal formation (25). To investigate whether the thinned cc resulted from defects in axon guidance, we examined glial development in P1 mice immunohistochemically with antiglial fibrillary acidic protein (anti-GFAP), a marker for astrocytes. Slightly enhanced glial differentiation was observed in the glial wedge (Figure 6, D-G) and indusium griseum (arrows and insets, Figure 6, D and E) of Tg newborns. Additionally, the transgene was expressed abundantly in cortical layers V and VI (Figure 6C), and cerebral cortex of $\mathrm{Tg}$ mice was significantly reduced in layer $\mathrm{V}$ 

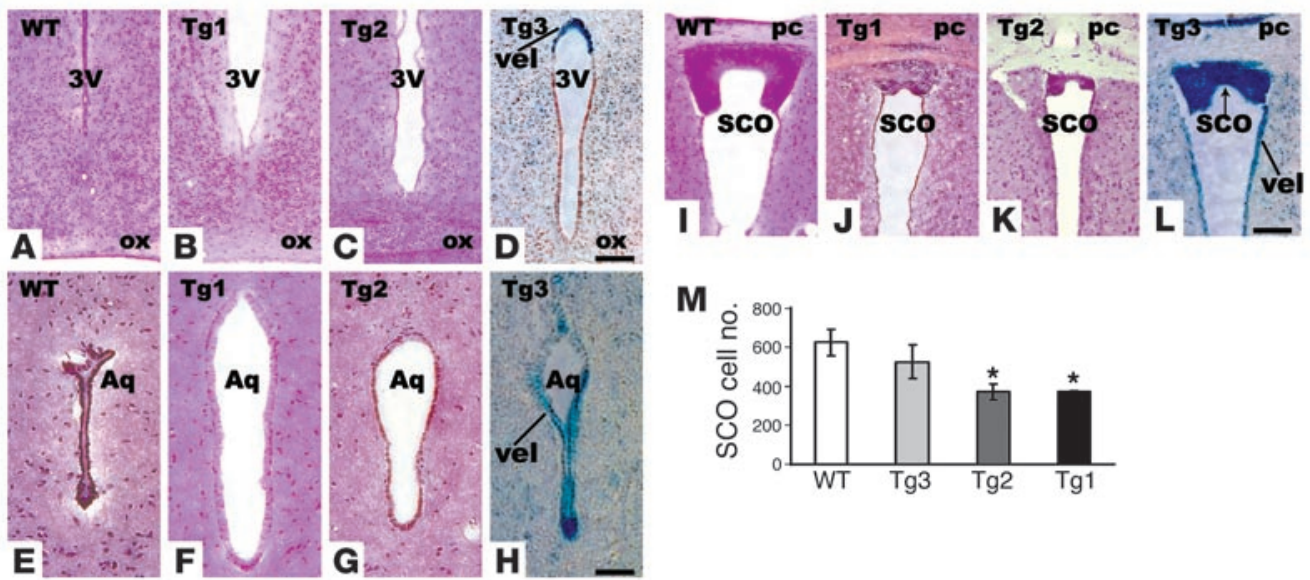

Figure 4

Reduced SCO and dilated third ventricle (3V) and aqueduct in Tg mice. (A-D) Coronal sections across the optic chiasm showed an enlarged third ventricle (3V) in each of the $3 \mathrm{Tg}$ lines (B-D) compared with their WT (A) littermates. (E-H) Comparison of coronal sections at the ventral tegmental decussation level displayed dilated aqueduct in all $3 \mathrm{Tg}$ lines $(\mathbf{F}-\mathbf{H})$, with a morphology grossly different from that in the WT littermates (E). (I-L) Images of coronal sections at the posterior commissure (pc) level revealed reduced SCO in all $3 \mathrm{Tg}$ lines $(\mathbf{J}-\mathbf{L})$ in comparison to the WT littermates (I). Sections in A-C, F, and I were stained with cresyl violet; sections in E, G, J, and $\mathbf{K}$ with H\&E; and sections in D, H, and L with $\mathbf{X}$-gal and neutral red. Note transgene expression in the ependymal cells (vel) of the third ventricle (D and $\mathbf{L})$ and aqueduct $(\mathbf{H})$ and in the SCO (arrow, L). (M) The SCO cell numbers at the pc level were significantly reduced in Tg2 and Tg1 mice. ${ }^{*} P<0.05$. Aq, aqueduct. Scale bars: 100 $\mu \mathrm{m}$ in $\mathbf{A}-\mathbf{D}$ and $\mathbf{I}-\mathbf{L} ; 50 \mu \mathrm{m}$ in $\mathbf{E}-\mathbf{H}$.

(Figure 6, A and B). These data suggest that reduced cortex and cc in Tg mice are largely due to lamination defects rather than compromised glial guidance of cortical axons.

Increased apoptosis and reduced proliferation in Tg embryos. The PAC1 mRNA (15) and our transgene were expressed intensely in proliferation zones of developing brain, indicating that PAC1 overexpression may interfere with neurogenesis. Previously, PACAP antagonists were reported to increase proliferation of cortical precursors in E14.5 rat embryos in vitro (26), while intraventricular administration of PACAP into E15.5 rat brains inhibited mitosis of cortical precursors (27).

To explore the cellular mechanisms of hydrocephalus-like abnormalities in PAC1-overexpressing mice, we analyzed neuronal proliferation and apoptosis by BrdU labeling and TUNEL assays. At mid-neurogenesis (E15.5), apoptosis was increased massively in the SCO (Figure 7, F and $\mathrm{H}$ ), the ventricular ependyma (arrowhead, Figure 7F), and the developing cortex (Figure 7, F and I). Within the cortex, apoptotic cells were intensively located in the cortical plate and marginal zone and to a much lesser degree in the proliferating zones (data not shown), indicating that the majority of apoptotic cells were postmitotic. Significantly, neuronal proliferation was also reduced in proliferating zones of cortex in $\mathrm{Tg}$ mice (Figure 7, A-D and J). However, at the early (E12.5) or late (E17.5)

\section{Figure 5}

Congenital brain abnormalities in PAC1 Tg mice. Nissl staining of serial coronal sections in a rostral-to-caudal direction from P1 WT (A, C, $\mathbf{E}$, and $\mathbf{G})$ and $\operatorname{Tg}(\mathbf{B}, \mathbf{D}, \mathbf{F}$, and $\mathbf{H})$ mice. Tg mice manifested a number of abnormalities, including dilated LVs, thinned cortex (Ctx), uncrossed $c c$ and formation of Probst bundles (p), widened longitudinal fissure between the 2 hemispheres (arrows), and rostralization together with underdevelopment of the hippocampus $(\mathrm{Hi})$. $\mathrm{CPu}$, caudate putamen; $\mathrm{MD}$, mediodorsal thalamus; ME, median eminence; mt, mammillothalamic tract; Sep, septum. stages of neurogenesis or in P1 brains, no significant difference in BrdU labeling was observed, nor was there a dramatic change in TUNEL labeling of P1 brains (data not shown).

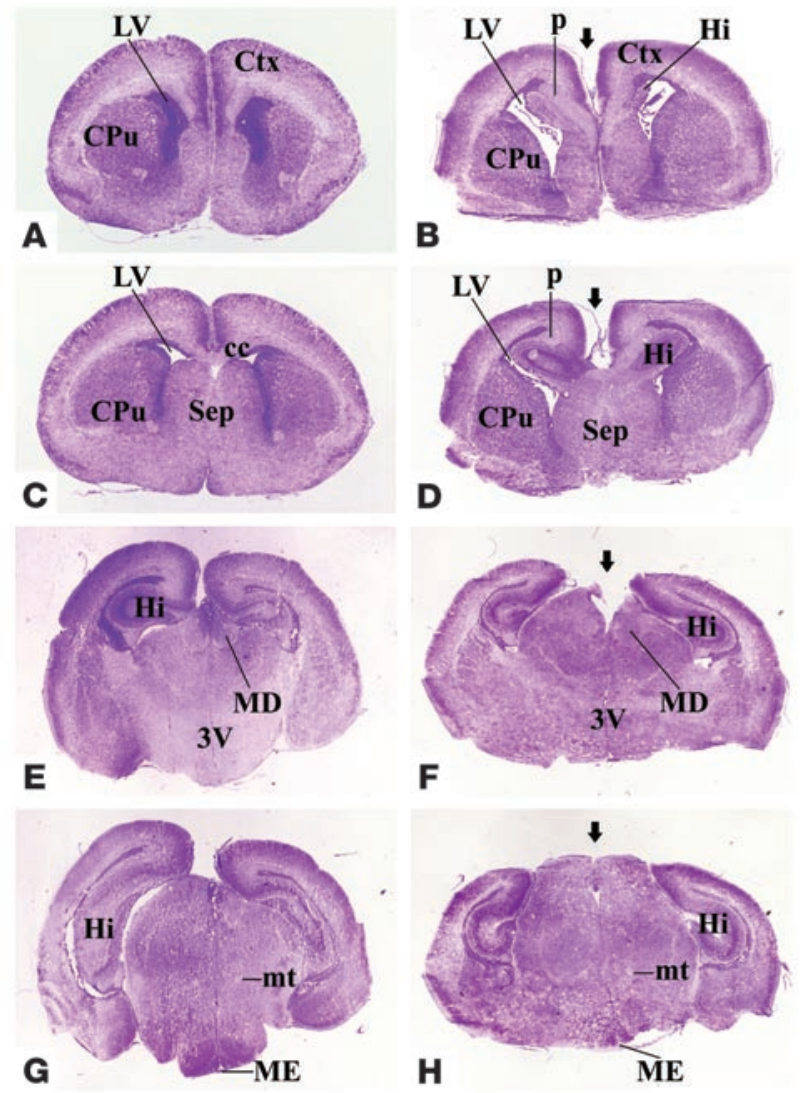




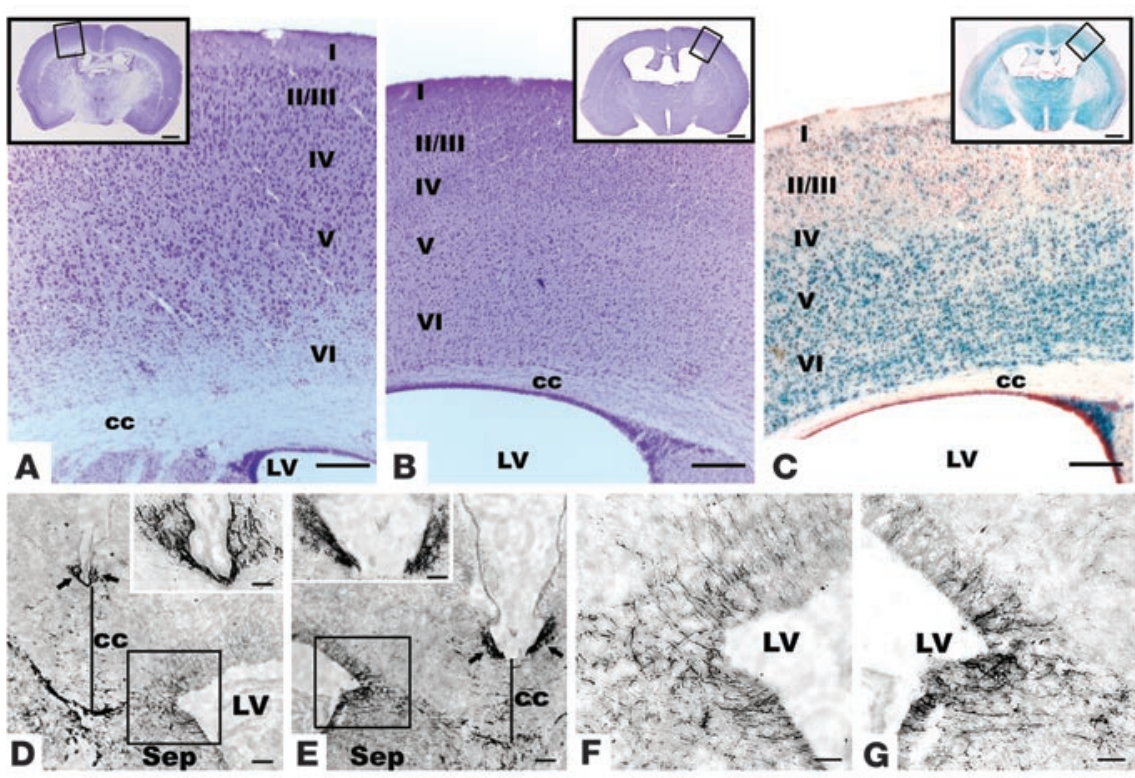

Figure 6

$\mathrm{Tg}$ mice are defective in cortical lamination but less likely defective in axonal guidance. (A and B) Nissl staining of coronal brain sections of WT (inset, A) and Tg (inset, B) littermates revealed dilated ventricles. Magnified images of the boxed regions shown in the insets of $\mathbf{A}$ and $\mathbf{B}$ illustrate thinned cerebral cortex and cc. Note that layer $\mathrm{V}$ was significantly reduced, with fewer large neurons (pyramidal). (C) X-gal staining showed transgene expression largely at layers $\mathrm{V}$ and VI. (D and $\mathbf{E}$ ) Glial fibrillary acidic protein (GFAP) immunostaining of brains of P1 WT mice (D) and Tg littermates (E) demonstrating a similar staining pattern at the glial wedge (black boxes) and the indusium griseum (arrows), magnified in the insets. Note that the thickness of cc (delineated by vertical lines) was also reduced in $\mathrm{Tg}$ newborns. $\mathbf{F}$ and $\mathbf{G}$ show magnification of the glial wedges delineated by the black boxes in $\mathbf{D}$ and $\mathbf{E}$, revealing slightly more glial staining in Tg mice (G). Scale bars: $200 \mu \mathrm{m}$ in A-E; $50 \mu \mathrm{m}$ in F and $\mathbf{G}$ and insets of $\mathbf{D}$ and $\mathbf{E} ; 1 \mathrm{~mm}$ in insets of $\mathbf{A}-\mathbf{C}$.

Reduced BrdU labeling may result from increased apoptosis. Therefore, we quantified BrdU-positive and total cells in a 0.01 $\mathrm{mm}^{2}$ area of E15.5 proliferating zones. Tg and WT embryos did not differ significantly in total cell numbers (WT, $316 \pm 15.6$ cells; Tg, $307 \pm 11.7$ cells; $n=5$ each; $P>0.05$; Figure 7J) or in cell density. However, the proportion of BrdU-labeled cells was reduced from $44 \%$ to $29 \%(n=5$ each; $P<0.01$; Figure $7 \mathrm{~K})$, suggesting that PAC1 overexpression caused a substantial proportion of neural progenitors to exit the mitotic phase, in harmony with previous reports $(26,27)$. Therefore, both reduced neuronal proliferation and increased apoptosis are associated with the hydrocephalusrelated defects in the Tg mice.

PAC1 overexpression affects neurogenesis rather than oligodendrocyte production. PACAP was shown to stimulate proliferation of oligodendrocytes in neonatal rats (28). To determine the effect of PAC1 overexpression on oligodendrocyte production in vivo, we immunolabeled E15.5 dorsal telencephalon with BrdU and PDGFR $\alpha$ (a marker for early oligodendrocyte progenitors [OLPs]). Weak staining of PDGFR $\alpha$ was found in both the ventricular and subventricular zones (Figure 8, C and D), but there was no dramatic difference in the cortex between Tg and WT embryos. Because of multiple origins of oligodendrocytes at different developmental stages and possible transdifferentiation of radial glia into oligodendrocytes after birth, we have examined P12 cortex (data not shown) and striatum (harboring abundant oligodendrocytes) with Sox10, a marker for later OLPs and mature oligodendrocytes, and observed no apparent difference between the WT and Tg mice (Figure 8, E and F). BrdUpositive cells were mainly located in the subventricular zone, and reduced proliferation in the cortex of Tg embryos correlated with expression of GLAST, a marker for radial glia (Figure 8, A and B). Collectively, these data indicate that reduced proliferation in the cortex of Tg embryos mainly affects ongoing neurogenesis rather than oligodendrocyte production.

Effects of PACAP on neurite outgrowth and neuronal migration in vitro. Thinner cerebral cortex and $\mathrm{cc}$ in the $\mathrm{Tg}$ mice may result from defects in neurite outgrowth and/or neuronal migration. To examine this possibility, we cultured primary neurons from E15.5 cortices of Tg and WT littermates in the absence (Figure 9, A and C) or presence of $10^{-7} \mathrm{M}$ PACAP (Figure 9, B and D) and measured neuronal migration in an 8-hour period (Figure 9E). Neurons from Tg embryos did not display defects in PACAP-induced neurite outgrowth (Figure 9D) compared with those from WT embryos (Figure 9B). In addition, the neurons from $\mathrm{Tg}$ migrated faster than those from WT embryos in the presence of PACAP (Figure 9E and Supplemental Video), suggesting that neurons of $\mathrm{Tg}$ embryos are unlikely to have major defects in neurite outgrowth or migration.

Disrupted cilia in ventricular ependyma of $\mathrm{Tg}$ mice. The PAC1 receptor is highly expressed in the ventricular ependymal cells (Figure 1, $\mathrm{I}, \mathrm{K}$, and $\mathrm{M}$ ), and coordinated beating of ependymal cilia is required to direct CSF flow. Cilia are highly structured, with microtubulebased large complexes known as intraflagellar transport (IFT) particles required for bidirectional movement of proteins between the base and the tip of cilia (29). An insertional mutation in a gene (Tg737) encoding Polaris, a component of the IFT particles, resulted in severe developmental defects, including hydrocephalus with disrupted cilia formation (30).

To examine whether PAC1 Tg mice have defects in the ventricular ependyma, we immunolabeled 5 -week brain sections with Abs against acetylated $\alpha$-tubulin and Polaris, key components of the IFT. WT ependymal cells were polarized, with oriented bundles of motile cilia of uniform length protruding into the LV lumen (red, Figure 10, A and B). In contrast, Tg mice had fewer cilia, and many of them were shorter, disorganized, and disorientated, as illustrated by anti-acetylated $\alpha$-tubulin staining (red, Figure 10, C-E). However, ependymal expression/localization of Polaris (Figure 10, A-E) and choroidal cilia (Figure 10, H and I) appeared normal in Tg mice. These data showed that hydrocephalus-like defects in PAC1 Tg mice are associated with severely disrupted ependymal cilia.

Elevated PKA/PKC signaling in brains of $\mathrm{Tg}$ mice. The signaling pathways involved in cilia formation are unknown. PAC1 receptor activation can trigger multiple signaling pathways, including PKA and PKC $(14,31)$. MARCKS is one of the first substrates characterized for PKC. Interestingly, MARCKS ${ }^{-/-}$mice exhibit dilated ventricles with complete agenesis of the cc and reduced cerebral 

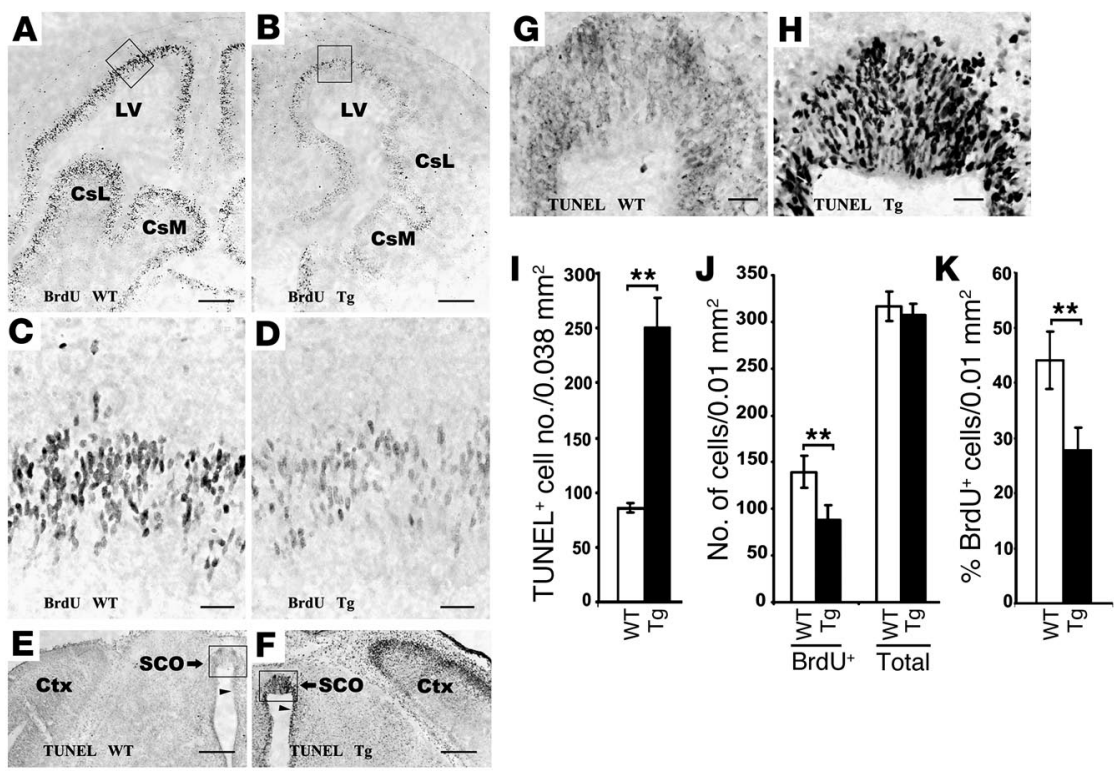

\section{Figure 7}

Decreased proliferation and increased apoptosis in E15.5 Tg embryos. (A-D) BrdU labeling of transverse sections of E15.5 Tg embryos and WT littermates. $\mathbf{C}$ and $\mathbf{D}$ are higher-power views of the boxed areas in $\mathbf{A}$ and $\mathbf{B}$, respectively. Note the substantial reduction in BrdU incorporation in Tg embryos. ( $E$ and $\mathbf{F}$ ) TUNEL assay of coronal sections of E15.5 WT (E) and $\mathrm{Tg}(\mathbf{F})$ embryos, demonstrating remarkable increases in apoptosis in the developing SCO (boxed areas, arrows), cortex (cortical plate and marginal zone in particular), and ventricular ependymal cells (arrowheads) in Tg embryos. ( $\mathbf{G}$ and $\mathbf{H}$ ) Higher magnification of the SCO in E and F. TUNEL-labeled cells (I) in E15.5 cortex were quantified from $0.038 \mathrm{~mm}^{2}$ of 3 sections of each embryo (WT, $n=4 ; \mathrm{Tg}, n=4)$. The number of BrdU-positive cells (J) or the total number of cells $(\mathbf{J})$ and their ratios $(\mathbf{K})$ within $0.01 \mathrm{~mm}^{2}$ of the proliferating zones of E15.5 cortex were quantified from 5 WT and $5 \mathrm{Tg}$ embryos, showing significantly reduced neuronal proliferation. ${ }^{* *} P<0.01$. CsL, corpus striatum laterale; CsM, corpus striatum mediale. Scale bars: $400 \mu \mathrm{m}$ in A, B, E, and F; $100 \mu \mathrm{m}$ in C, D, G, and H.

cortex (5). CREB is a well-characterized substrate for PKA, and $\mathrm{CREB}^{-/-}$mice show enlarged LVs with severely reduced $\mathrm{cc}$ and anterior commissures (10).

To determine changes of PKA/PKC activity in Tg mice, we immunolabeled brain sections with $\mathrm{Ab}$ against phosphorylated CREB (phospho-CREB). In agreement with elevated PACAP signaling, phospho-CREB was significantly increased in ependymal nuclei of Tg mice (Figure 10, F and G). To quantify PKA/PKC activity, we analyzed levels of phospho-CREB and phospho-MARCKS by immunoblotting. As anticipated, PAC1 overexpression did not alter the total level of MARCKS expression (Figure 11B). However, phospho-MARCKS was increased significantly in brains of Tg mice and correlated with transgene dosage (Figure 11B). Interestingly, both total CREB and phospho-CREB were increased in brains of Tg mice (Figure 11A). Our results, together with previously published data from $C R E B^{-/-}$and $M A R C K S^{-/-}$mutants, suggest that both gain- and loss-of-function in PKA/PKC signaling may lead to hydrocephalus-related manifestations.

\section{Discussion}

We have developed Tg mice overexpressing the human PAC1 receptor. Tg mice showed marked reductions in the cerebral cortex, cc, and SCO together with decreased neuronal proliferation and accelerated apoptosis. Consistent with receptor signaling, both PKA and PKC activity were increased in brains of Tg mice.
Ventricular ependyma of $\mathrm{Tg}$ mice displayed elevated phospho-CREB immunoreactivity and disorganized cilia. Most importantly, Tg mice developed transgene dosage-dependent hydrocephalus with dilated third and LVs. These results constitute the first clear evidence to our knowledge that GPCRs can be involved in hydrocephalus-like neurodevelopmental disorders.

Hydrocephalus and ependymal cilia. CSF is mainly produced by choroid plexus, and ventricular ependymal cilia are vital for directing CSF flow. Crucially, abnormal cilia are found in patients with primary ciliary dyskinesia, a condition known to be associated with congenital heart disease and/or hydrocephalus (32). Within cilia, bidirectional movement of proteins is mediated through the microtubule-based IFT particles (29). In mice, mutations in key components (Polaris, Spag6, Hydin, or Mdnah) of IFT all result in hydrocephalus with impaired ependymal cilia function (30, 33-35). The PAC1 transgene is highly expressed throughout the ventricular ependyma. One possibility, therefore, is that PAC1 overexpression affects ependymal cilia via cytoskeleton-associated molecules. Indeed, ependymal cilia of $\mathrm{Tg}$ mice were fewer in number, shorter, and disorientated compared with WT. On the other hand, we detected no significant change of cilia in choroid plexus, consistent with an extremely low level of the transgene expression. These data indicate that disrupted ependymal cilia at least partially contribute to progressive hydrocephalus in PAC1 Tg mice. Our preliminary observation suggests that CSF flow is affected in the Tg mice (data not shown).

Hydrocephalus and neuronal proliferation/apoptosis. Apoptosis was implicated in the development of hydrocephalus of Msx 1 mutants (24). However, hyperproliferation of neural progenitor cells was also associated with severe hydrocephaly in $\mathrm{Lgl1}^{-/}$mice (36). We demonstrated remarkably reduced proliferation in the developing cortex, SCO, and ventricular ependyma of E15.5 Tg embryos, consistent with the previous observation that intraventricular delivery of PACAP transiently inhibited mitosis in E15.5 rat brains (27). We also showed for the first time that PAC1 overexpression significantly increased apoptosis, which was not apparent following intraventricular delivery of PACAP (27), and this might be due to rapid degradation of injected PACAP in vivo (37) or because prolonged elevation of PACAP signaling is required to trigger apoptosis.

Thinned cortex and $\mathrm{cc}$ can arise from aborted neurogenesis if newborn neurons have defects in neurite outgrowth or fail to extend their axons to the targeted destination. Our data show that neurite outgrowth or neuronal migration is not compromised in Tg mice. Astrocytes at the glial wedge and the indusium griseum are critical for guiding axons to cross the midline (25). However, they are well developed in Tg newborns. On the other hand, we observed a dramatic reduction in cortical layer $V$, where a large proportion of callosal neurons reside. Therefore, the 

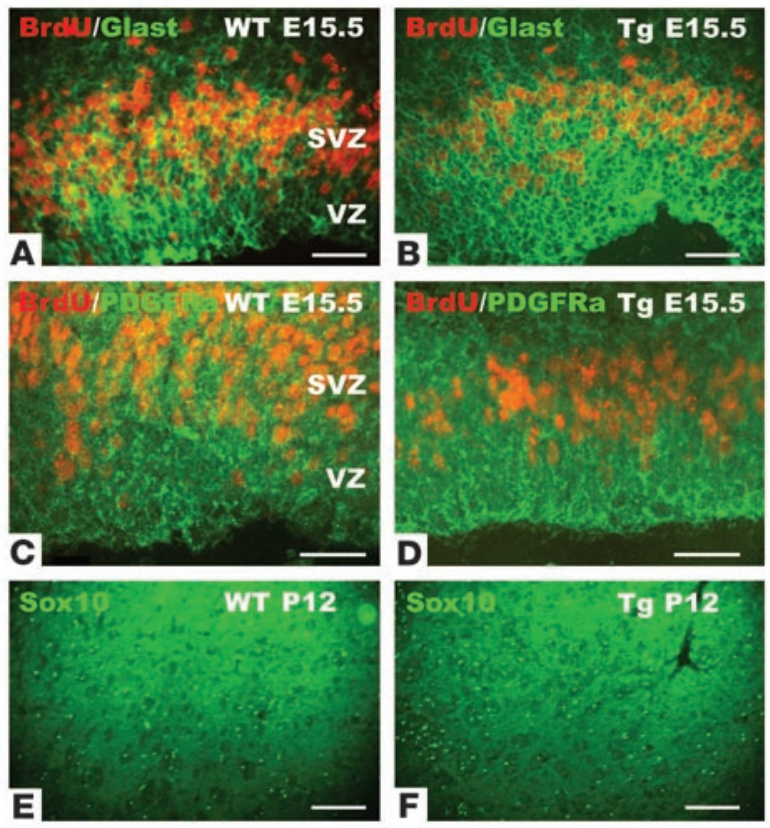

thinned $\mathrm{cc}$ or failure of $\mathrm{cc}$ to cross the midline in $\mathrm{Tg}$ newborns is likely to result from defects in cortical lamination rather than in astrocyte differentiation.

A reduced number of oligodendrocytes may also cause thinning of the cerebral cortex and/or cc. Recent studies revealed 3 waves of OLP production in the developing forebrain, and a normal brain can be developed with a normal complement of oligodendrocytes and myelin, even if any of the 3 OLP populations is destroyed (38). In line with this discovery, there were no apparent changes in PDGFR $\alpha$ expression at E15.5 in the cortex of $\mathrm{Tg}$ embryos; nor was there an overt alteration of Sox 10 expression in P12 mice. Additionally, the normal appearance of anterior and posterior commissures in Tg adults also excludes the possibility of abnormal oligodendrocyte production. Therefore, defects in cerebral cortex and cc of $\mathrm{Tg}$ mice are unlikely due to defects in oligodendrocyte production.

Hydrocephalus and posttranslational modifications. Socs7 regulates receptor tyrosine kinases, and deficiency of Socs7 causes hydrocephalus $(1,2)$. Hydrocephalus-related phenotypes are observed in mutants lacking PKC substrates (4-6) or PKA (9). However, elevated cAMP production also causes hydrocephalus in $\mathrm{Tg} 737$ mice (30). We have shown that PKA/PKC activity is increased in brains of Tg mice.

\section{Figure 8}

Neurogenesis but not oligodendrocyte generation is affected by PAC1 overexpression. (A and B) Double immunostaining of E15.5 cortex with BrdU (red) and GLAST (green) showed reduced BrdU labeling in the subventricular zone (SVZ) of developing cortex of Tg embryos (B) compared with that in the WT control (A). Note that most, if not all, of the BrdU-labeled cells coexpressed GLAST. (C and D) Colabeling of E15.5 cortex with BrdU and PDGFR $\alpha$. Cells in the ventricular zone (VZ) and SVZ at this stage were weakly stained with anti-PDGFR $\alpha$, indicating they are multipotential neural stem cells. No significant difference in PDGFR $\alpha$ staining was observed at E15.5 in cortex of WT and Tg embryos. (E and F) Immunostaining of Sox10 showed comparable labeling of Sox10-positive cells in P12 striatum of WT (E) and Tg (F) littermates. Scale bars: $50 \mu \mathrm{m}$ in A-F.

Crucially, phospho-CREB is upregulated in ventricular ependymal cells, and cilia are disorganized in Tg mice. These data suggest that appropriate levels of PKA/PKC signaling are essential for normal ventricular function and that disruption of $\mathrm{PKA} / \mathrm{PKC}$ signaling in ependymal cells may underpin a hydrocephalic phenotype.

PAC1 Tg and PAC1 $1^{-1-}$ mice. Gene dosage is crucial for normal brain development and physiological function. Often overdose and underdose cause similar abnormalities, and this is true for the PAC1 receptor. The PAC1-overexpressing and $\mathrm{PAC1} 1^{-/}$mice displayed strikingly similar phenotypes. First, pre-weaning loss is common in $P A C 1^{-/-}$(39), $P A C A P^{-/-}$(40), and our Tg mice. Second, both $P A C 1^{-/-}$(39) and PAC1 Tg (data not shown) neonates rapidly develop pulmonary hypertension-related complications. Third, PAC1 ${ }^{-/}$mutants show altered response to photic stimulation (41), and circadian function is also modulated in PAC1 Tg mice (data not shown). Fourth, PAC1-overexpressing and $\mathrm{PAC1}^{-/-}$(data not shown) newborns displayed similar brain abnormalities.

It is increasingly recognized that many human diseases do not result from deletions or mutations in coding sequences but instead are associated with disruption of long-distance regulatory elements (42). In this respect, the Tg and knockout technologies are complementary in illustrating gene function. In the H-Tx rat, 2 of the susceptibility loci for hydrocephaly are mapped to chromosome 4q24-42 and 9q36-38 (43), where genes encoding rat PAC1 receptor and PACAP are localized, respectively. In patients, elevated PACAP signaling may contribute to CNS abnormalities $(16,17,19)$. We have shown that altered levels of PACAP signaling are associated with hydrocephalus in mice. Therefore, misregulation or mutations in genes encoding PACAP and/or the PAC1 receptor could be associated with hydrocephalus-related neurodevelopmental disorders in mice, rats, and humans.

\section{Figure 9}

PACAP promotes neurite outgrowth and migration in neurons of $\mathrm{Tg}$ embryos. At E15.5, neurons from developing cortex of $\mathrm{Tg}$ or WT embryos were cultured on $35-\mathrm{mm}$ dishes at a density of $10^{4}$ cells $/ \mathrm{ml}$ in the absence (A and $\mathbf{C}$ ) or presence (B and $\left.\mathbf{D}\right)$ of PACAP $\left(10^{-7} \mathrm{M}\right)$ for 4 hours prior to recording. Migrational behavior was recorded with time-lapse imaging for the subsequent 8 hours, and migration rates of 100-120 cells under each condition from 3 independent experiments were quantified by 1-way ANOVA $\left({ }^{\star} P<0.05\right)($ E). Note that (a) PACAP promotes neurite outgrowth in neurons of both WT and Tg embryos (B and D) and (b) PACAP significantly increases the migration rate of neurons of Tg embryos (E).
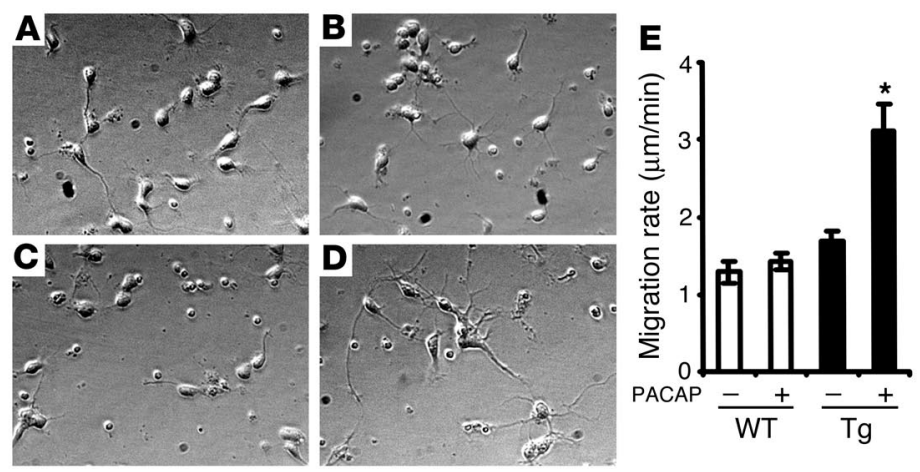

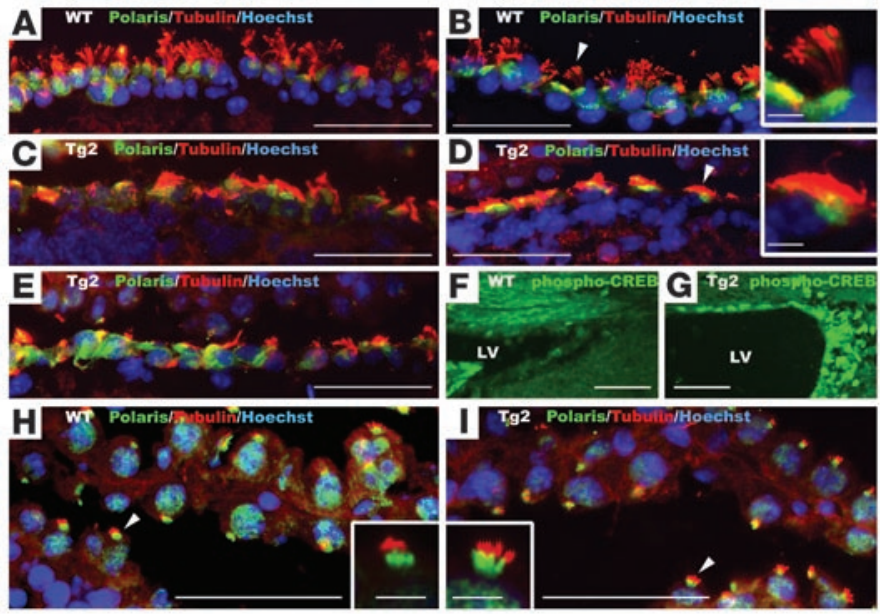

\section{Figure 10}

Disorganized cilia in ventricular ependyma but not in choroid plexus of Tg mice. Brains from three 5-week-old Tg2 mice (C-E, G, and I) and 2 WT (A, B, F, and H) littermates were coronally sectioned. Sections were costained with Hoechst (blue), anti-acetylated $\alpha$-tubulin (red), and anti-Polaris (green) $(\mathbf{A}-\mathbf{E}, \mathbf{H}$, and I) or stained with anti-phospho-CREB ( $\mathbf{F}$ and $\mathbf{G}$ ). Insets in $\mathbf{B}, \mathbf{D}, \mathbf{H}$, and $\mathbf{I}$ are magnified views of cilia (arrowheads) in the respective panels. Note that ependyma of $\mathrm{Tg}$ mice exhibited disorganized cilia and increased phospho-CREB reactivity. Scale bars: $50 \mu \mathrm{m}$ in A-I and $5 \mu \mathrm{m}$ in insets of $\mathbf{B}, \mathbf{D}, \mathbf{H}$, and $\mathbf{I}$.

\section{Methods}

PAC clones. PAC clones were obtained by hybridization of the RPCI-1 Human PAC Library (21) and analyzed by FISH, restriction mapping, pulsed-field electrophoresis, Southern blotting, STS mapping, PCR, sequencing, and bioinformatics (Basic Local Alignment Search Tool [BLAST], http://www.ncbi.nlm.nih.gov/BLAST/; UCSC Human Genome Browser, http://genome.ucsc.edu/cgi-bin/hgGateway; and GeneJockey II, Biosoft).

Targeted modification. We have adopted the bacterial artificial chromosome modification protocol (20) for introducing an IRES-lacZ reporter gene into 204D22, with a 791-bp HindIII fragment and the adjacent 1.6-kb HindIII-SacI fragment flanking the PAC1 stop codon as homologous recombination arms. A 4-kb IRES-lacZ-polyA cassette was inserted between the 2 arms. The 7.4-kb homologous recombination cassette was reexcised with BamHI-SalI and cloned into the PSV1 vector. The final construct was transformed into 204D22, and correct homologous recombinants were identified by Southern hybridization.

Generation of $\mathrm{Tg}$ mice. All experimental procedures were conducted in accordance with the United Kingdom Animals (Scientific Procedures) Act of 1986 and were approved by the Ethical Review Committee, University of Aberdeen, and the Home Office (London, United Kingdom). Tg founders were produced as described before (12) with NotI-digested PAC DNA and characterized using 6 pairs of transgene-specific PCR primers. The transgene copy number was determined by semiquantitative PCR of Het genomic DNA, with primers PAC1For (5'-CAGGATTATTACTACCTGTC$\left.3^{\prime}\right)$ and PAC1Rev (5'-GGAAGAAGGTCTCCACCAGC-3') at 14-24 cycles with Expand Long Template System (Roche Diagnostics) using NIH Image software (version 1.61; http://rsb.info.nih.gov/nih-image/).

\section{Figure 11}

Elevated PKA and PKC activity in PAC1-overexpressing mice. Proteins were extracted from brains of WT (lane 1), Tg3 (lane 2), Tg2 (lane 3), and Tg1 (lane 4) mice. (A) One hundred micrograms of total proteins were resolved by SDS-PAGE and sequentially probed with Abs against phospho-CREB ( $p$-CREB), total CREB, and tubulin. (B) Thirtyfive micrograms of proteins were run on SDS-PAGE and sequentially immunoblotted with Abs against phospho-MARCKS (p-MARCKS), total MARCKS, and tubulin. The relative abundance of proteins CREB and phospho-CREB (C) or MARCKS and phospho-MARCKS (D) was quantified from 4 independent immunoblots.
Expression of the transgene. After genotyping with yolk sac DNA, RNA was extracted from individual E10.5 embryos using RNAzol B (Biogenesis). RT was carried out with $1 \mu \mathrm{g}$ total RNA using Omniscript kits (QIAGEN) at $37^{\circ} \mathrm{C}$ for 1 hour. RT-PCR was performed with primers PAC1For and PAC1Rev for 30 cycles. PCR products were digested with RsaI and resolved on agarose gels. $\mathrm{X}$-gal staining of embryos was done as described previously (12).

Tissue preparation and histological analysis. Adult mice were humanely killed with a lethal dose of sodium pentobarbitone and perfused transcardially with cold paraformaldehyde (4\%). Newborn mice were killed with sodium pentobarbitone and immersed in the fixative for 4 hours. E12.5 and E15.5 embryos were dissected and fixed for 2 hours. After cryoprotection with $15 \%$ sucrose in PBS overnight, serial coronal brain sections or transverse embryo sections at $12 \mu \mathrm{m}$ were processed in a cryostat (CM1850; Leica Microsystems) and mounted on Polysine slides (VWR). Sections were stained with cresyl violet, $\mathrm{H} \& \mathrm{E}$, neutral red, oil red O, X-gal, or Abs (see below).

BrdU labeling and TUNEL assay. Time-mated pregnant females were killed 50 minutes after BrdU injection (i.p., $50 \mathrm{mg} / \mathrm{kg}$ body weight). Embryos were fixed and sectioned as described above. For BrdU immunostaining, sections were treated with $0.4 \%$ pepsin (Sigma-Aldrich) in PBS for 30 minutes at $37^{\circ} \mathrm{C}$, denatured with $2 \mathrm{~N} \mathrm{HCl}$ for 30 minutes at $37^{\circ} \mathrm{C}$, and neutralized with $0.1 \mathrm{M}$ sodium borate ( $\mathrm{pH} 8.5$ ) for 10 minutes at room temperature. $\mathrm{BrdU}$ incorporation assays (BD Biosciences) and TUNEL assays (Roche Diagnostics) were executed according to the manufacturers' instructions.

Immunohistochemistry. Sections were immunohistochemically processed as described previously (44). The primary Abs were mouse anti-BrdU (1:200; BD Biosciences) and rabbit anti-GFAP (1:1,000; Dako). The secondary Abs included biotinylated goat anti-mouse or anti-rabbit secondary Ab (1:400; Sigma-Aldrich). Immunofluorescence staining was carried out with primary
A

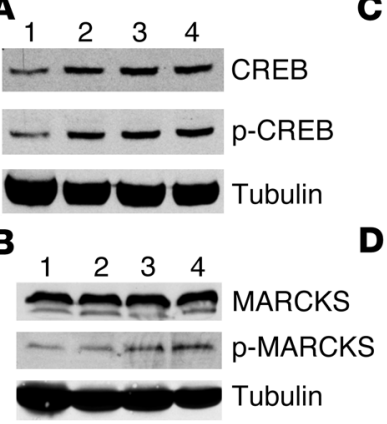

D $\frac{.}{\bar{D}}$

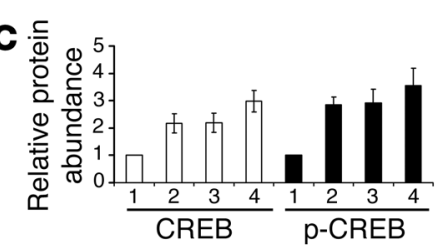


Abs guinea pig anti-GLAST (1:4,000; Chemicon International), guinea pig anti-Sox10 (1:2,000; kindly provided by Michael Wegner, University Medical Center Hamburg-Eppendorf, Hamburg, Germany), rabbit anti-PDGFR $\alpha$ (1:100, Abcam), rabbit anti-Polaris (1:500; a gift from Bradley K. Yoder, University of Alabama at Birmingham, Birmingham, Alabama, USA), rabbit anti-phospho-CREB (1:200; Upstate USA Inc.), and mouse anti-acetylated $\alpha$-tubulin (1:1,500; Sigma-Aldrich). The secondary Abs were FITC-conjugated donkey anti-guinea pig IgG (1:200; Jackson ImmunoResearch Laboratories Inc.), FITC-conjugated donkey anti-rabbit IgG (1:400; Invitrogen), and Texas red-conjugated donkey anti-mouse IgG (1:1,000; Invitrogen).

Immunoblotting. Soluble proteins from whole-brain homogenates $(35 \mu \mathrm{g}$ for MARCKS or $100 \mu \mathrm{g}$ for CREB) were resolved by SDS-PAGE and transferred to nitrocellulose membranes (Bio-Rad). The membranes were immunoblotted with primary Abs goat anti-MARCKS (1:5,000; Santa Cruz Biotechnology Inc.), rabbit anti-phospho-MARCKS (Ser152/156) (1:250; Cell Signaling Technology), rabbit anti-CREB (1:750; Cell Signaling Technology), rabbit anti-phospho-CREB (1:200; Upstate USA Inc.), and mouse anti-acetylated $\alpha$-tubulin (1:500,000; Sigma-Aldrich), followed by secondary Abs donkey anti-goat IgG HRP-linked Ab (1:3,000; Santa Cruz Biotechnology Inc.), goat anti-rabbit IgG HRP-linked Ab (1:3,000; New England Biolabs Inc.), and goat anti-mouse IgG (Fc specific)-peroxidase Ab (1:20,000; SigmaAldrich). The immunoreactive bands were visualized using ECL (SigmaAldrich) and quantified with an imaging densitometer (Bio-Rad GS-690).

Migration assay. E15.5 primary neurons were prepared as described previously (44) at a density of $10^{4}$ cells $/ \mathrm{ml}$ in neurobasal medium (Invitrogen) supplemented with $2 \%$ B27 (Invitrogen) and $2 \mathrm{mM}$ glutamine (SigmaAldrich). Three milliliters of cells were plated per $35-\mathrm{mm}$ dish precoated with poly-D-lysine and cultured at $37^{\circ} \mathrm{C}$ with $5 \% \mathrm{CO}_{2}$. Cytosine arabinoside $(10 \mu \mathrm{M})$ was added 24 hours after plating to prevent proliferation of non- neuronal cells. PACAP ${ }_{1-38}\left(10^{-7} \mathrm{M}\right)$ was added 4 hours before and during time-lapse recording, and the recording was carried out in $\mathrm{CO}_{2}$-independent medium (Invitrogen) supplemented with $2 \mathrm{mM}$ glutamine and $15 \%$ FCS. Neuronal migration was time-lapse recorded on MetaMorph (Universal Imaging Corp.) and a Digital Pixel imaging system (Digital Pixel) in a temperature-controlled chamber. Data analysis was processed with MetaMorph software.

Statistics. Images were captured by an Axiovert 40CFL microscope and quantified with AxioVision Rel. 4.5 software (Zeiss). Data were analyzed by 1 -way ANOVA and are presented as mean $\pm \mathrm{SD}$. $P<0.05$ was considered to be statistically significant.

\section{Acknowledgments}

We acknowledge the Scottish Hospital Endowments Research Trust, Chief Scientist Office of the Scottish Executive, BDF Newlife, University of Aberdeen, and NHS Grampian for funding the project. We thank Michael Wegner for anti-Sox10 and Bradley K. Yoder for anti-Polaris. We also thank Nathaniel Heintz (Rockefeller University, New York, New York, USA) for the pSV1 vector and for the homologous recombination protocol.

Received for publication December 6, 2005, and accepted in revised form April 25, 2006.

Address correspondence to: Sanbing Shen, School of Medical Sciences, College of Life Sciences and Medicine, University of Aberdeen, Institute of Medical Sciences, Foresterhill, AB25 2ZD Aberdeen, United Kingdom. Phone: 44-1224-555730; Fax: 441224-555719; E-mail: sanbing.shen@abdn.ac.uk.
1. Krebs, D.L., et al. 2004. Development of hydrocephalus in mice lacking SOCS7. Proc. Natl. Acad. Sci. U. S. A. 101:15446-15451.

2. Banks, A.S., et al. 2005. Deletion of SOCS7 leads to enhanced insulin action and enlarged islets of Langerhans. J. Clin. Invest. 115:2462-2471. doi:10.1172/JCI23853.

3. Galarza, M. 2002. Evidence of the subcommissural organ in humans and its association with hydrocephalus. Neurosurg. Rev. 25:205-215.

4. Chen, J., et al. 1996. Disruption of the MacMARCKS gene prevents cranial neural tube closure and results in anencephaly. Proc. Natl. Acad. Sci. U. S. A. 93:6275-6279.

5. Wu, M., Chen, D.F., Sasaoka, T., and Tonegawa, S. 1996. Neural tube defects and abnormal brain development in F52-deficient mice. Proc. Natl. Acad. Sci. U. S. A. 93:2110-2115.

6. Brouns, M.R., et al. 2000. The adhesion signaling molecule $\mathrm{p} 190$ RhoGAP is required for morphogenetic processes in neural development. Development. 127:4891-4903.

7. Cogram, P., Hynes, A., Dunlevy, L.P., Greene, N.D., and Copp, A.J. 2004. Specific isoforms of protein kinase $\mathrm{C}$ are essential for prevention of folateresistant neural tube defects by inositol. Hum. Mol. Genet. 13:7-14.

8. Loeken, M.R. 2005. Current perspectives on the causes of neural tube defects resulting from diabetic pregnancy. Am. J. Med. Genet. C. Semin. Med. Genet. 135:77-87.

9. Huang, Y., Roelink, H., and McKnight, G.S. 2002. Protein kinase A deficiency causes axially localized neural tube defects in mice. J. Biol. Chem. 277:19889-19896.

10. Rudolph, D., et al. 1998. Impaired fetal T cell development and perinatal lethality in mice lacking the cAMP response element binding protein. Proc. Natl. Acad. Sci. U. S. A. 95:4481-4486.
11. Harmar, A.J., et al. 1998. International union of pharmacology. XVIII. Nomenclature of receptors for vasoactive intestinal peptide and pituitary adenylate cyclase-activating polypeptide. Pharmacol. Rev. 50:265-270.

12. Shen, S., et al. 2000. Overexpression of the human VPAC2 receptor in the suprachiasmatic nucleus alters the circadian phenotype of mice. Proc. Natl. Acad. Sci.U. S. A. 97:11575-11580.

13. Harmar, A.J., et al. 2002. The VPAC(2) receptor is essential for circadian function in the mouse suprachiasmatic nuclei. Cell. 109:497-508.

14. Spengler, D., et al. 1993. Differential signal transduction by five splice variants of the PACAP receptor. Nature. 365:170-175.

15. Waschek, J.A., et al. 1998. Neural tube expression of pituitary adenylate cyclase-activating peptide (PACAP) and receptor: potential role in patterning and neurogenesis. Proc. Natl. Acad. Sci. U. S. A. 95:9602-9607.

16. Salihu, H.M., Boos, R., and Schmidt, W. 1997. Antenatally detectable markers for the diagnosis of autosomally trisomic fetuses in at-risk pregnancies. Am. J. Perinatol. 14:257-261.

17. Takeda, K., Okamura, T., and Hasegawa, T. 1989. Sibs with tetrasomy $18 \mathrm{p}$ born to a mother with trisomy 18p. J. Med. Genet. 26:195-197.

18. Faraone, S.V., et al. 2005. Genome scan of schizophrenia families in a large Veterans Affairs Cooperative Study sample: evidence for linkage to $18 \mathrm{p} 11.32$ and for racial heterogeneity on chromosomes 6 and 14. Am. J. Med. Genet. B. Neuropsychiatr. Genet. 139:91-100.

19. Miller, M., Kaufman, G., Reed, G., Bilenker, R., and Schinzel, A. 1979. Familial, balanced insertional translocation of chromosome 7 leading to offspring with deletion and duplication of the inserted segment, $7 \mathrm{p} 15$ leads to $7 \mathrm{p} 21$. Am. J. Med. Genet. 4:323-332.
20. Yang, X.W., Model, P., and Heintz, N. 1997. Homologous recombination based modification in Escherichia coli and germline transmission in Tg mice of a bacterial artificial chromosome. Nat. Biotechnol. 15:859-865.

21. Ioannou, P.A., et al. 1994. A new bacteriophage P1derived vector for the propagation of large human DNA fragments. Nat. Genet. 6:84-89.

22. Louvi, A., and Wassef, M. 2000. Ectopic engrailed 1 expression in the dorsal midline causes cell death, abnormal differentiation of circumventricular organs and errors in axonal pathfinding. Development. 127:4061-4071.

23. Blackshear, P.J., et al. 2003. Graded phenotypic response to partial and complete deficiency of a brain-specific transcript variant of the winged helix transcription factor RFX4. Development. 130:4539-4552.

24. Ramos, C., Fernandez-Llebrez, P., Bach, A., Robert, B., and Soriano, E. 2004. Msx1 disruption leads to diencephalon defects and hydrocephalus. Dev. Dyn. 230:446-460.

25. Shu, T., and Richards, L.J. 2001. Cortical axon guidance by the glial wedge during the development of the corpus callosum. J. Neurosci. 21:2749-2758.

26. Carey, R.G., Li, B., and DiCicco-Bloom, E. 2002. Pituitary adenylate cyclase activating polypeptide anti-mitogenic signaling in cerebral cortical progenitors is regulated by p57Kip2-dependent CDK2 activity. J. Neurosci. 22:1583-1591.

27. Suh, J., Lu, N., Nicot, A., Tatsuno, I., and DiCiccoBloom, E. 2001. PACAP is an anti-mitogenic signal in developing cerebral cortex. Nat. Neurosci. 4:123-124.

28. Lee, M., et al. 2001. Pituitary adenylyl cyclase-activating polypeptide stimulates DNA synthesis but delays maturation of oligodendrocyte progenitors. J. Neurosci. 21:3849-3859.

29. Scholey, J.M. 2003. Intraflagellar transport. Annu. Rev. Cell Dev. Biol. 19:423-443. 
30. Banizs, B., et al. 2005. Dysfunctional cilia lead to altered ependyma and choroid plexus function, and result in the formation of hydrocephalus. Development. 132:5329-5339.

31. Nicot, A., Lelievre, V., Tam, J., Waschek, J.A., and DiCicco-Bloom, E. 2002. Pituitary adenylate cyclase-activating polypeptide and sonic hedgehog interact to control cerebellar granule precursor cell proliferation. J. Neurosci. 22:9244-9254.

32. Bush, A. 2000. Primary ciliary dyskinesia. Acta Otorhinolaryngol. Belg. 54:317-324.

33. Sapiro, R., et al. 2002. Male infertility, impaired sperm motility, and hydrocephalus in mice deficient in sperm-associated antigen 6. Mol. Cell. Biol. 22:6298-6305.

34. Davy, B.E., and Robinson, M.L. 2003. Congenital hydrocephalus in hy 3 mice is caused by a frameshift mutation in Hydin, a large novel gene. Hum. Mol. Genet. 12:1163-1170.

35. Ibanez-Tallon, I., et al. 2004. Dysfunction of axonemal dynein heavy chain Mdnah5 inhibits ependymal flow and reveals a novel mechanism for hydrocephalus formation. Hum. Mol. Genet. 13:2133-2141.

36. Klezovitch, O., Fernandez, T.E., Tapscott, S.J., and Vasioukhin, V. 2004. Loss of cell polarity causes severe brain dysplasia in Lgl1 knockout mice. Genes Dev. 18:559-571.

37. Zhu, L., et al. 2003. The role of dipeptidyl peptidase IV in the cleavage of glucagon family peptides: in vivo metabolism of pituitary adenylate cyclase activating polypeptide-(1-38). J. Biol. Chem. 278:22418-22423.

38. Kessaris, N., et al. 2006. Competing waves of oligodendrocytes in the forebrain and postnatal elimination of an embryonic lineage. Nat. Neurosci. 9:173-179.

39. Otto, C., et al. 2004. Pulmonary hypertension and right heart failure in pituitary adenylate cyclaseactivating polypeptide type I receptor-deficient mice. Circulation. 110:3245-3251.
40. Hashimoto, H., et al. 2001. Altered psychomotor behaviors in mice lacking pituitary adenylate cyclase-activating polypeptide (PACAP). Proc. Natl. Acad. Sci. U. S. A. 98:13355-13360.

41. Hannibal, J., et al. 2001. Dissociation between lightinduced phase shift of the circadian rhythm and clock gene expression in mice lacking the pituitary adenylate cyclase activating polypeptide type 1 receptor. J. Neurosci. 21:4883-4890.

42. Kleinjan, D.A., and van Heyningen, V. 2005. Longrange control of gene expression: emerging mechanisms and disruption in disease. Am.J. Hum. Genet. 76:8-32.

43. Jones, H.C., Carter, B.J., Depelteau, J.S., Roman, M., and Morel, L. 2001. Chromosomal linkage associated with disease severity in the hydrocephalic H-Tx rat. Behav. Genet. 31:101-111.

44. Lang, B., et al. 2004. Astrocytes in injured adult rat spinal cord may acquire the potential of neural stem cells. Neuroscience. 128:775-783. 